\title{
Propiedades de adsorción en zeolitas con anillos de 8 miembros I. Microporosidad y superficie externa
}

\author{
CORONA ${ }^{\mathrm{I}}$, O.L.; HERNÁNDEZ ${ }^{\mathrm{I}}$, M.A.; HERNÁNDEZ ${ }^{\mathrm{I}}$, F.; ROJAS ${ }^{\mathrm{II}}$, F.; PORTILLO ${ }^{\mathrm{III}}$, R.; LARA ${ }^{\mathrm{II}}$, V.H.; \\ CARLOS $^{\text {IV }}$, F.M. \\ ${ }^{\text {I }}$ Departamento de Investigación en Zeolitas, Instituto de Ciencias de la Universidad Autónoma de Puebla, \\ México. Edificio 76, Complejo de Ciencias, Ciudad Universitaria, CP 72570, Puebla, Pue. México \\ e-mail: leobardo02@hotmail.com; mighern@siu.buap.mx; fhernand@siu.buap.mx \\ II Departamento de Química, Universidad Autónoma Metropolitana-Iztapalapa \\ e-mail: fernando@xanum.uam.mx; lacv@xanum.uam.mx \\ III Facultad de Ciencias Químicas, Universidad Autónoma de Puebla \\ e-mail: portilloreyes@yahoo.com \\ IV Fisicoquímica de materiales, ICUAP \\ e-mail: carlos.felipe@icbuap.buap.mx
}

\section{RESUMEN}

En este trabajo se reporta la síntesis de zeolita LTA y sus posteriores intercambios iónicos con distintas sales inorgánicas. De manera adicional se reportan datos experimentales sobre la estimación del área externa en zeolita LTA intercambiada usando el método $t$. La caracterización físico-química de estas zeolitas es abordada a través de métodos de Difracción de Rayos X (DRX), Microscopía Electrónica de Barrido (SEM), Espectroscopía de Infrarrojo (IR) y Adsorción de $\mathrm{N}_{2}$ a $76 \mathrm{~K}$. La zeolita LTA fue sintetizada hidrotérmicamente partiendo de una mezcla compuesta por hidróxido de sodio, aluminato de sodio y silicato de sodio en las relaciones estequiométricas requeridas. Posteriormente se le realizó un intercambió iónico con nitrato de plata y sales en forma de cloruros de $\mathrm{K}, \mathrm{Ca}, \mathrm{Cd}, \mathrm{Zn}, \mathrm{Cu}, \mathrm{y} \mathrm{Mn}$. La zeolita intercambiada con cationes bivalentes, presentó mejores propiedades para la adsorción de nitrógeno. Los espectros de rayos $\mathrm{X}$ de la zeolita matriz y de aquella intercambiada mostraron buena cristalinidad. La microscopía electrónica de barrido mostró la presencia de un gran número de cristales definidos y biselados. El estudio de caracterización de textura de la zeolita intercambiada con las distintas sales mencionadas mediante la adsorción de nitrógeno a $76 \mathrm{~K}$ mostró que es posible obtener substratos de zeolita LTA que desarrollen altas porosidades. La mayoría de los microporos de estos materiales son supermicroporos (huecos entre 0.7 y $2 \mathrm{~nm}$ ). El valor de la microporosidad determinada por el método $\alpha$ de Sing para la muestra intercambiada con Ca, revela que los microporos contenidos en la zeolita incluyen ultramicroporos y supermicroporos, en los cuales se lleva a cabo el llenado primario y secundario respectivamente.

Palabras clave: microporos, zeolitas, área externa, zeolita LTA, adsorción.

\section{Adsorption properties in zeolites containing 8-membered rings I. Microporosity and external surface area}

\section{ABSTRACT}

In this work synthesis of LTA zeolite and its ionic exchange with inorganic chemical salts were studied. Likewise experimental results are reported about LTA zeolite external surface area by using the t-plot method. Physicochemical characterization of this zeolite was carried out by XRD, SEM, IR and nitrogen adsorption (76 K) techniques. LTA zeolite was synthesized hydrothermally starting from a hydrogel mixture composed by $\mathrm{NaOH}, \mathrm{Na}[\mathrm{Al}(\mathrm{OH}) 4]$ and $\mathrm{Na} 2 \mathrm{SiO} 4$ in stoichiometric amounts determined by its crystallization field. An ion exchange was carried out in LTA zeolite with AgNO3, $\mathrm{KCl}, \mathrm{CaCl} 2, \mathrm{CdCl} 2, \mathrm{ZnCl} 2, \mathrm{CuCl} 2$ and $\mathrm{MnCl} 2(0.1 \mathrm{~N}$ for 
all of them). The LTA zeolite exchanged with bivalent cations showed the best properties to nitrogen adsorption. X-Ray diffraction showed good crystallization.

Scanning electronic microscopy showed very well defined crystals existence. Textural studies of exchanged zeolite with chemical salts mentioned before by nitrogen adsorption method indicated that it is possible to obtain LTA zeolite substrates which contain high porosity. A great number of micropores of these zeolites are supermicropores ( $0.7-2 \mathrm{~nm}$, pore-diameter). Microporosity studies by as-plots of Sing was carry out and revealed that in LTA zeolite exchanged with $\mathrm{CaCl} 2$ exist ultramicropores and supermicropores in which the primary and secondary filling is carried out respectively.

Keywords: micropores, zeolites, external area, LTA zeolite, adsorption.

\section{INTRODUÇÃO}

Las zeolitas son materiales microporosos [diámetro de poro $(w), w<2 \mathrm{~nm}$ ], IUPAC, 1985 [1] cristalinos constituidos principalmente de átomos de Si y Al (llamados átomos T), cada uno de los cuales esta unido de forma tetraédrica a los átomos de O. Debido a que este tipo de materiales son cristalinos, presentan distribuciones de tamaño de poro estrechas. Considerando este aspecto, las zeolitas presentan muchas aplicaciones donde el tamaño y la selectividad de forma son indispensables, tales como catálisis, intercambio iónico y adsorción. Los poros en las zeolitas son clasificados por el número de átomos $\mathrm{T}$ contenidos en los anillos que rodean a los poros. Las zeolitas que contienen poros limitados por anillos de 8, 10 y 12 átomos $\mathrm{T}$ son consideradas como zeolitas de poro pequeño, mediano y grande. Por ejemplo, la zeolita LTA contiene anillos de 8 miembros y genera poros del orden de 4-5 Å., Curtis et al., 2003 [2]].

Un ejemplo representativo de la aplicación de este tipo de zeolita se refiere a su uso como intercambiador iónico. En la literatura científica se menciona que el $67 \%$ de las zeolitas producidas a nivel mundial, se consumen como zeolitas Tipo A o Linde Tipo A (LTA), como componente de los detergentes. Su función es fijar "iones duros" $\left(\mathrm{Mg}^{2+} \mathrm{o} \mathrm{Ca}^{2+}\right)$ del agua de lavado para que no impidan la acción de los surfactantes, Chang, 2000 [3]].

En México, la mayor parte de los detergentes que se encuentran en el mercado contienen polifosfatos en lugar de zeolitas LTA y no existe alguna regulación formulada que prohíba su venta, Hernández, 2000 [4]. Este tipo de zeolita tiene una gran capacidad de intercambio para los iones bivalentes como el $\mathrm{Ca}^{2+} \mathrm{y} \mathrm{Mg}^{2+}$. La red cristalina de la zeolita LTA, se puede describir en términos de dos tipos de poliedros; uno compuesto por un arreglo cúbico sencillo de ocho tetraedros y otro es un octaedro truncado constituido por 24 tetraedros. La celda unitaria de la zeolita LTA contiene 24 tetraedros: 12 de $\mathrm{AlO}_{4}$ y 12 de $\mathrm{SiO}_{4}$. Cuando la zeolita esta totalmente hidratada contiene 27 moléculas de $\mathrm{H}_{2} \mathrm{O}$. La zeolita A se sintetiza en la forma sódica y el contenido de su celda unitaria es: $\mathrm{Na}_{12}\left[\mathrm{Al}_{12} \mathrm{Si}_{12} \mathrm{O}_{48}\right]$.W $\mathrm{H}_{2} \mathrm{O}$; W es un número entero, Chintansinh, 2005 [ㄷ]. Las zeolitas LTA presentan una estructura porosa de tres dimensiones 3-D, con poros que corren en forma perpendicular uno al otro en los ejes $a, b$ y $c$. El diámetro de poro de la zeolita NaA está definido por anillos de oxígeno de 8 miembros con dimensiones del orden de $0.43 \mathrm{~nm}$, Figura 1. La variación de los tamaños de poros esta definida por el tipo de catión a intercambiar debido a que en esta zeolita han sido identificados tres sitios para los cationes: Los sitios SI, SII y SIII.

Una de las propiedades más importantes de este tipo de zeolita es su selectividad de forma, la cual es controlada en forma primaria por su estructura microporosa. Sin embargo, la selectividad de forma total de una zeolita dada puede ser significativamente afectada por procesos no selectivos que suceden en el área externa ( $A e)$ de la zeolita en cuestión. La gran mayoría de las zeolitas sintéticas están constituidas de microporos $(w<2 \mathrm{~nm})$ y presentan, asimismo, áreas externas bajas que pueden ser evaluadas por las áreas geométricas aparentes utilizando directamente la microscopía electrónica de barrido (SEM, por sus siglas en inglés). Sin embargo, frecuentemente muchas zeolitas no son perfectamente cristalinas y pueden exhibir mesoporosidad $(2<w<50$ $\mathrm{nm})$ y, consecuentemente presentan áreas externas mucho más altas que sus áreas geométricas aparentes. Diversas técnicas han sido aplicadas para evaluar el área externa Ae de las zeolitas, pero la gran mayoría de los investigadores usan la adsorción de $\mathrm{N}_{2}$ a $77 \mathrm{~K}$ utilizando el método $t$ o el método $\alpha_{\mathrm{s}}$. Algunos autores utilizan estos métodos para evaluar estas propiedades en un grupo de zeolitas ZSM-5, ERI, MOR y FAU, Cejka et al., 2002 []] , Carrot et al., 2005 [7], Sing et al. 1985 [8]. El éxito en la aplicación de estos dos métodos se basa en la elección adecuada de un sólido de referencia o estándar con un área conocida, Jaroniec, 1999 [9]]. La segunda técnica sugerida para este propósito consiste en bloquear los microporos presentes con adsorbatos adecuados (neopentano, n-nonano), antes de realizar las estimaciones de las áreas específicas disponibles o restantes por el 
tradicional método BET, utilizando $\mathrm{N}_{2}$ a $77 \mathrm{~K}$, Roberts et al., 1987 [10]. El uso del método $t$ para la estimación de $A e$ en sólidos microporosos es muy conocido y ha sido aplicado con bastante éxito a sólidos constituidos con microporos, Remy et al., 1995 [11].

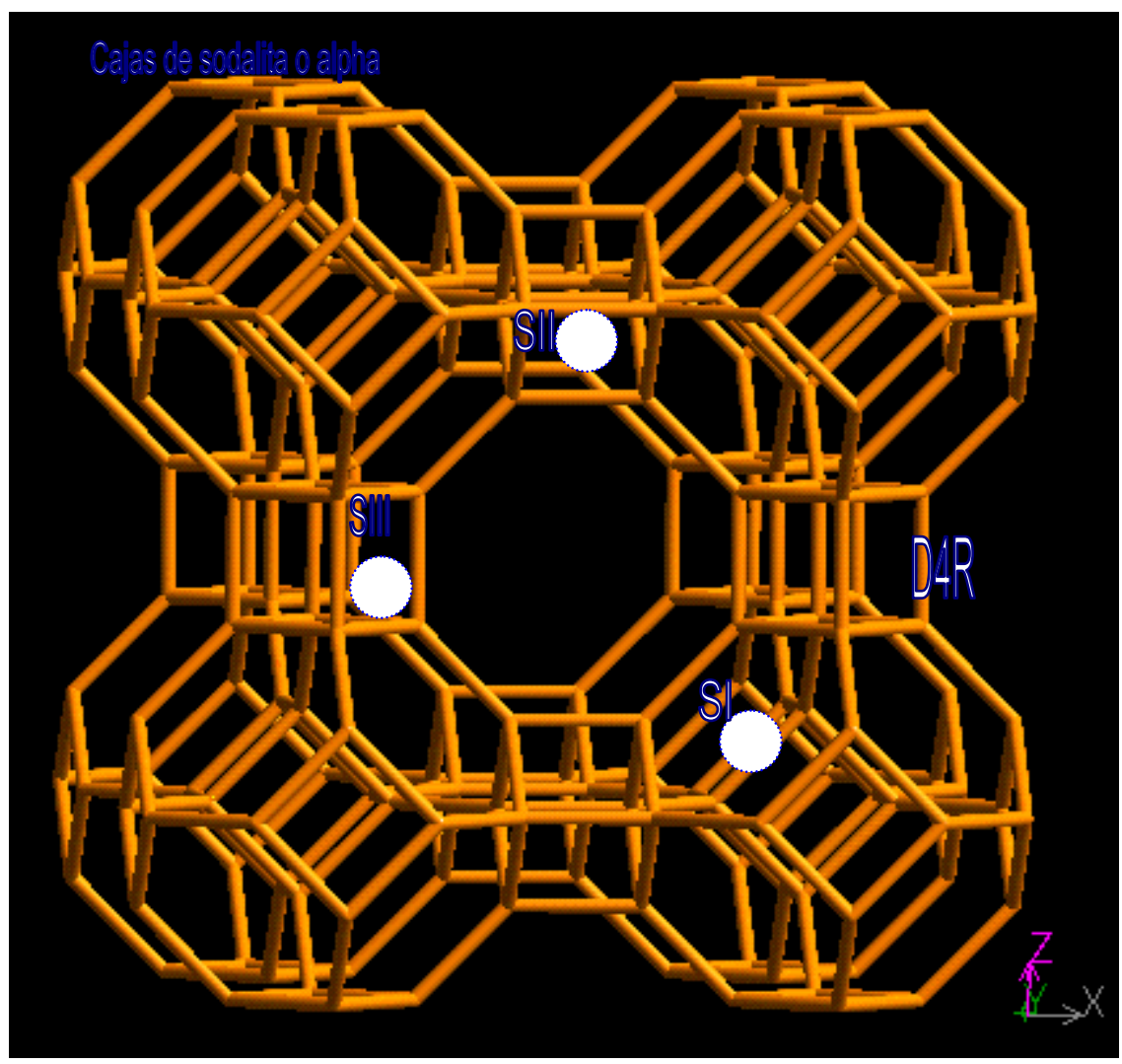

Figura 1: Estructura de la zeolita LTA (IZA).

En este trabajo, se reportan datos experimentales sobre la estimación de área externa y volumen de microporos en zeolitas LTA. También se reporta la síntesis de este tipo de zeolita y sus posteriores intercambios iónicos con distintas sales inorgánicas. La caracterización físico-química de estas zeolitas es abordada a través de métodos de Difracción de Rayos X, Microscopía Electrónica de Barrido, Espectroscopia de Infrarrojo y Adsorción de $\mathrm{N}_{2}$ a $76 \mathrm{~K}$.

El manuscrito esta organizado de la siguiente manera: Los detalles experimentales del trabajo son proporcionados de manera inmediata, éstos incluyen: los materiales y métodos empleados, la síntesis de la zeolita, los intercambios iónicos, la descripción y condiciones experimentales de las técnicas de caracterización y las mediciones de las isotermas de adsorción de $\mathrm{N}_{2}$ en un sistema volumétrico de adsorción. Posteriormente, distintos métodos de análisis de los parámetros de la estructura porosa son brevemente descritos para calcular importantes parámetros estructurales, una discusión de los resultados de caracterización es realizada, y finalmente, las conclusiones más importantes de este trabajo son contempladas.

\section{PARTE EXPERIMENTAL}

\subsection{Materiales}

Hidróxido de sodio, $\mathrm{NaOH}$, Hidróxido de aluminio, $\mathrm{Al}(\mathrm{OH})_{3}$, cloruro de potasio, $\mathrm{KCl}$, cloruro de calcio, $\mathrm{CaCl}_{2}$, cloruro de cadmio, $\mathrm{CdCl}_{2}$, cloruro de manganeso, $\mathrm{MnCl}_{2}$, cloruro de cobre, $\mathrm{CuCl}$, y cloruro de zinc, $\mathrm{ZnCl}_{2}$, grado analítico (> 99.998\%) suministrados por Aldrich fueron usados para la síntesis e intercambio iónico 
de la zeolita LTA. Helio (He) y nitrógeno $\left(\mathrm{N}_{2}\right)$ de alta pureza (> 99.99\% suministrados por LINDE) fueron seleccionados para realizar los estudios de textura de la zeolita LTA intercambiada.

\subsection{Síntesis de zeolita LTA}

Zeolita Linde Tipo A (LTA) fue sintetizada a partir una mezcla de hidrogeles puros de hidróxido de sodio, $\mathrm{NaOH}$, aluminato de sodio, $\mathrm{Na}\left[\mathrm{Al}(\mathrm{OH})_{4}\right]$ y metasilicato de sodio, $\mathrm{Na}_{2} \mathrm{SiO}_{3}$, en cantidades estequiométricas determinadas por los campos de cristalización. El proceso de síntesis de la zeolita LTA consiste en un crecimiento cristalino en fase coloidal que se lleva a cabo a temperatura y tiempo de reacción que dependen del tipo de estructura que se desee obtener. Para este tipo de zeolita la síntesis inicia con una mezcla de las geles reactantes en un sistema cerrado bajo agitación a temperaturas cercanas a $30^{\circ} \mathrm{C}$. A la temperatura del orden de $95^{\circ} \mathrm{C}$ se realiza un proceso de sembrado de cristales de la estructura a conseguir (tipo LTA), y posteriormente los cristales crecen durante algunas horas (24 horas para la zeolita LTA) bajo temperatura y agitación. La zeolita matriz LTA, se intercambió con nitrato de plata y sales en forma de cloruros $\left(\mathrm{KCl}, \mathrm{CaCl}_{2}, \mathrm{CdCl}_{2}, \mathrm{ZnCl}_{2}, \mathrm{CuCl} 2\right.$, $\mathrm{MnCl}_{2}$ ) colocando en un matraz $5 \mathrm{~g}$ de esta zeolita matriz y $500 \mathrm{~mL}$ de agua destilada. Por otro lado, se preparó una solución de $100 \mathrm{~mL}$ conteniendo $0.5 \mathrm{~g}$ de cada una de las sales químicas cada vez, la cual se adicionó lentamente al sistema de reacción manteniéndolo bajo agitación a una temperatura de $85^{\circ} \mathrm{C}$ durante $24 \mathrm{hrs}$. Las zeolitas obtenidas fueron etiquetadas como: NaA, CaNaA, KNaA, CdNaA, ZnNaA, CuNaA, MnNaA y AgNaA. La mesoporosidad de estos sólidos se atribuye a fases que no alcanzaron a cristalizar durante el periodo de síntesis. La porosidad total en estos sólidos cristalinos es alta (50- 80\%). En la Tabla 1 están listados los valores de propiedades físicas de los metales a intercambiar en las zeolitas LTA.

Tabla 1: Radio iónico de metales a intercambiar en zeolitas LTA.

\begin{tabular}{ccc}
\hline Catión & $\begin{array}{c}\text { Radio Iónico } \\
\AA\end{array}$ & Tipo de Orbítales \\
& 1.26 & $5 \mathrm{~S} 1$ \\
$\mathrm{Ag}+$ & 1.95 & $3 \mathrm{~S} 1$ \\
$\mathrm{Na}^{+}$ & 1.33 & $4 \mathrm{~S} 1$ \\
$\mathrm{~K}+$ & & $4 \mathrm{~S} 2$ \\
$\mathrm{Mn} 2+$ & 0.96 & $4 \mathrm{~S} 1$ \\
$\mathrm{Cu} 2+$ & 0.74 & $4 \mathrm{~S} 2$ \\
$\mathrm{Zn} 2+$ & 0.97 & $5 \mathrm{~S} 2$ \\
$\mathrm{Cd} 2+$ & 0.99 & $4 \mathrm{~S} 2$ \\
\hline $\mathrm{Ca} 2+$ & & \\
\hline
\end{tabular}

\subsection{Mediciones}

Los patrones de difracción de Rayos X fueron obtenidos usando un difractómetro Siemens D-500. Los compuestos presentes en las muestras sometidas a estudio se identificaron con ayuda de tarjetas del Comité Conjunto de Estándares de Difracción (JCPDS). La morfología y el tamaño de los cristales fueron observados usando un microscopio electrónico Vega Tescan TS5136SB dotado de un procesador de imágenes de alta resolución. La Espectroscopía de Infrarrojo de las zeolitas estudiadas se realizó en un Espectrómetro Nicolet 5ZDX, en el intervalo de espectro de $4000-400 \mathrm{~cm}^{-1}$, el detector es del tipo MCT. Todas las isotermas de adsorción de $\mathrm{N}_{2}$ fueron medidas a la temperatura de ebullición del nitrógeno líquido (76 K en la ciudad de Puebla, México) en un sistema de adsorción volumétrico automático (ASC-1 de Quantachrome).

\subsection{Métodos de cálculo}

La superficie específica de la zeolita LTA intercambiada fue estimada usando las ecuaciones BET, Brunauer, 2005 [12] y Langmuir, Ruthven, 1984 [13], en el intervalo de presiones relativas $p / p^{0}=0.04-0.2$. El volumen total de poros $\left(\mathrm{V}_{\Sigma}\right)$ fue estimado a la $p / p^{0}=0.95$, evaluado como volumen de líquido. El volumen de microporo $\left(\mathrm{W}_{0}\right)$ fue calculado usando los gráficos $\alpha_{s}$ de Sing, Roberts et al., 1987 [10]. 


\subsection{1 Área externa (Ae)}

Para calcular el grosor estadístico $t(\mathrm{~nm})$ de la capa adsorbida de $\mathrm{N}_{2}$ como una función de la $p / p^{0}$ la ecuación de De Boer puede ser usada, Huai, 2004 [14]:

$t\left(p / p^{0}\right)(n m)=\left[\frac{13.99}{0.0434-\log \left(p / p^{0}\right)}\right]^{1 / 2}$

Este tipo de gráficos y los de Harkins Jura son usados para calcular el volumen de microporos y área externa a partir de datos experimentales de adsorción de $\mathrm{N}_{2}$ a $77 \mathrm{~K}$. Para adsorbentes constituidos con microporos, los gráficos $t$ (la representación del grosor estadístico de la capa adsorbida vs. volumen adsorbido) proporcionan el establecimiento de diferentes zonas, cada una de ellas asociadas a un mecanismo específico: a) La zona inicial, representa el desarrollo de una capa adsorbida en los mesoporos y microporos amplios, b) La etapa intermedia, asociada al crecimiento progresivo del grosor de la capa adsorbida $t$ en todos los poros presentes, excepto los que se llenaron inicialmente (etapa a) y finalmente c) La etapa final; ésta representa la adsorción en la superficie externa de los materiales sometidos a estudio, Hudec, 2002 [1]․ A partir de esta etapa, los datos experimentales de adsorción son evaluados a través de la siguiente ecuación:

$$
A_{e}=15.47 \frac{v}{t}
$$

\section{RESULTADOS Y DISCUSIÓN}

\subsection{Caracterización}

\subsubsection{Difracción de rayos $\mathrm{X}$}

En la Figura 2 se observan los patrones de difracción de Rayos X de las zeolitas LTA estudiadas. De esta figura se observa que en el patrón de rayos $\mathrm{X}$ correspondiente a la zeolita CuNaA se observan señales poco definidas atribuidas a que durante el tratamiento de intercambio iónico con $\mathrm{CuCl}_{2}$ se están formando nuevas fases cristalinas de cloruros con otros cationes $\left(\mathrm{Na}^{+}\right)$presentes en el sólido precursor NaA. La estructura cristalina de la zeolita LTA permanece inalterable después de los tratamientos de intercambio iónico con las distintas sales restantes como ha sido demostrado por la presencia de todas las señales correspondientes a la zeolita LTA. De estos patrones de difracción se observan señales características a $2 \theta=7^{\circ}, 10^{\circ}, 12.5^{\circ}, 22^{\circ}, 23.5^{\circ}, 27^{\circ}, 30^{\circ}$ y $34^{\circ}$, típicas para zeolita de estas características; patrones de difracción con comportamiento similar han sido reportados previamente, Chudasama et al., 2005 [్]. 


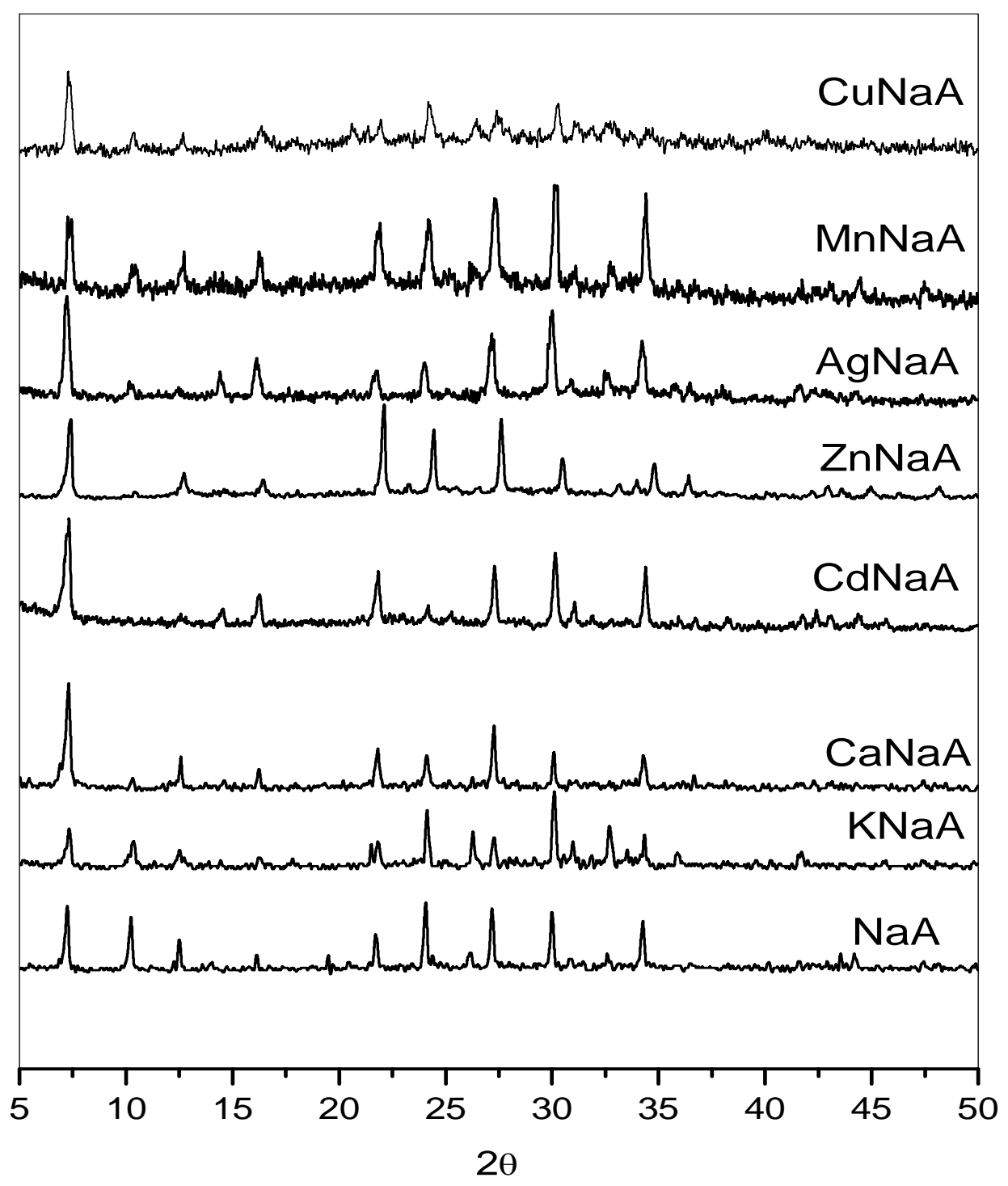

Figura 2: Patrones de difracción de rayos X de zeolitas LTA intercambiadas.

Con base en este esquema es de suponer que el proceso de intercambio iónico en los mesoporos, macroporos y área externa del sólido, se realizó en forma geométricamente irregular, es decir que, como resultado del proceso de intercambio iónico, se formaron agrupaciones (clusters) de los cationes intercambiados. Este efecto condujo a que al aumentar la cantidad de moléculas adsorbidas, éstas interaccionaran entre sí, provocando un incremento en la energía total de interacción, ver DRX en muestras CuNaA, y AgNaA.

\subsubsection{Microscopía electrónica de barrido}

La microscopía electrónica de barrido (MEB) muestra que la forma de los cristales obtenidos de la zeolita AgNaA en la síntesis presenta una simetría cúbica, con aristas definidas y biseladas, Figura 3. De esta figura se observa que los cristales poseen dimensiones cercanas a $2 \mu \mathrm{m}$. La micrografía obtenida fue amplificada 10000 y 30000 veces respecto a su tamaño original. Cristales de estas dimensiones proporcionan ventajas en estos materiales al presentar considerables áreas externas debido que reducen la resistencia a la transferencia de la masa. Estas propiedades hacen que estas zeolitas sean deseables para muchos procesos industriales como es el caso de los intercambios iónicos. 
(a)

(b)

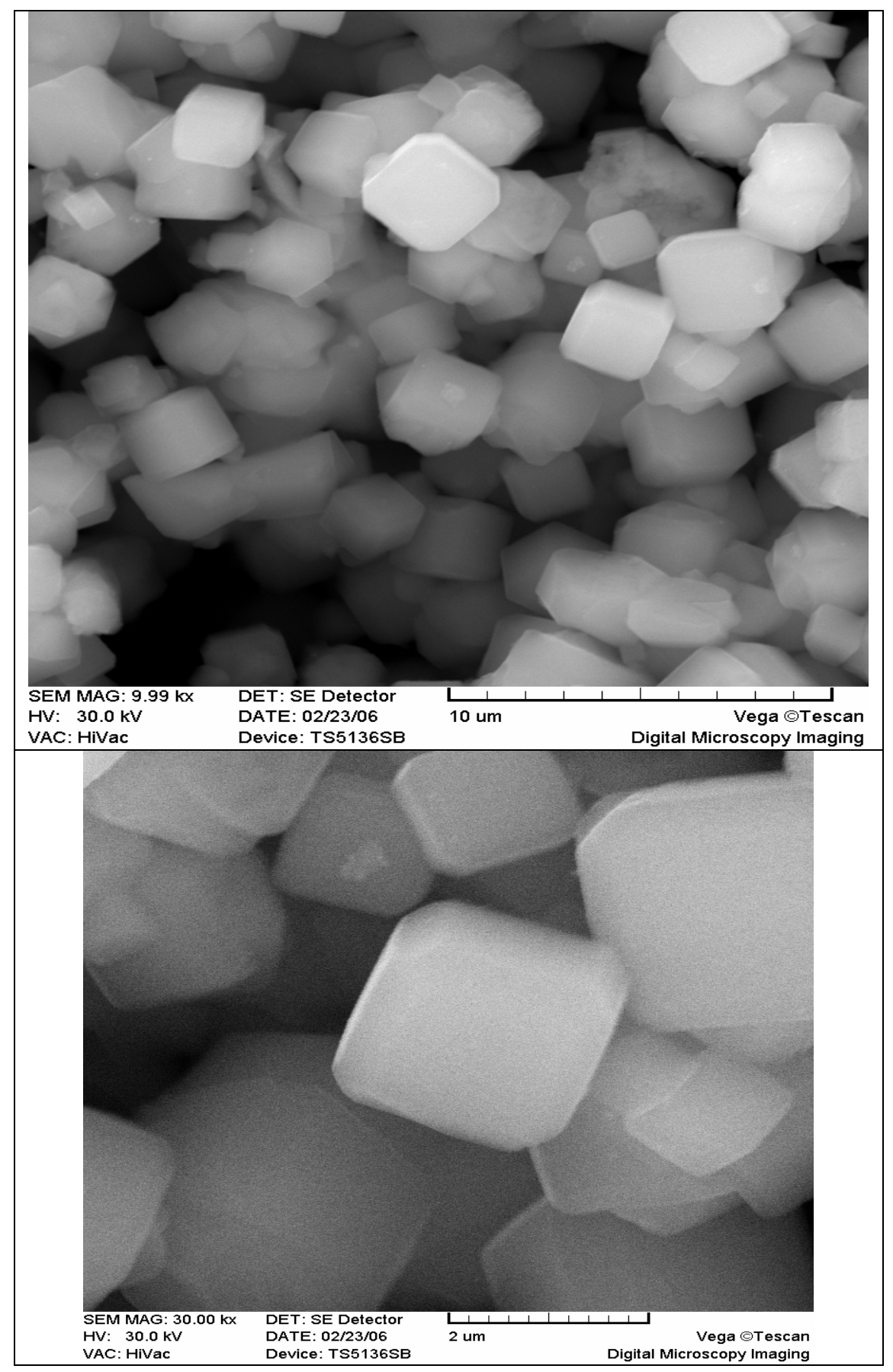

Figura 3: MEB de zeolita NaA. a) AgNaA amplificada 10000 veces, b) 30000 veces

\subsubsection{Espectroscopia de infrarrojo}

Los espectros IR de las zeolitas LTA intercambiadas se muestran en la Figura 4(a) De esta figura se observa que las señales prominentes de este tipo de materiales se encuentran en el intervalo en número de onda 4000-200 $\mathrm{cm}^{-1}$. Las señales resultantes provienen de la absorción de frecuencias definidas de estas zeolitas y exhiben las vibraciones de tensión (estiramiento o "stretching") de los puentes de $\mathrm{H}$ entre el agua adsorbida físicamente que se encuentra en las cavidades y los oxígenos superficiales. Este tipo de señales dan origen a una 
banda entre 3400-3700 $\mathrm{cm}^{-1}$ que se traslapa con las vibraciones de tensión de los hidroxilos en la capa octaédrica. Este tipo de interacción contempla la vibración de tensión del $\mathrm{OH}$ unido a aluminios octaédricos $\left(\mathrm{Al}_{2} \mathrm{OH}\right)$ con su carga negativa exacerbada por la proximidad de una sustitución de $\mathrm{Al}$ por un metal $\left(\mathrm{M}^{+}\right)$. Así como las vibraciones de tensión y de deformación de los $\mathrm{H}-\mathrm{O}-\mathrm{H}$ correspondientes al agua molecular adsorbida. Las vibraciones de tensión en el plano de los Si-O-Si de la capa tetraédrica, dan generalmente fuertes bandas de absorción entre 970 y $1070 \mathrm{~cm}^{-1}$ que para el caso de esta zeolita LTA aparece aproximadamente a los $1000 \mathrm{~cm}^{-1}$. Sin embargo, las vibraciones de deformación ocasionan bandas débiles entre 400-500 $\mathrm{cm}^{-1}$ siendo la zona de 490 $540 \mathrm{~cm}^{-1}$ particularmente sensible, donde su frecuencia varía inversamente con el radio iónico de los cationes octaédricos. En la Figura 4b se observan las vibraciones del enrejado de las zeolitas LTA, finalmente, en la Figura 4c se observa las vibraciones Si-OH de las zeolitas LTA estudiadas.
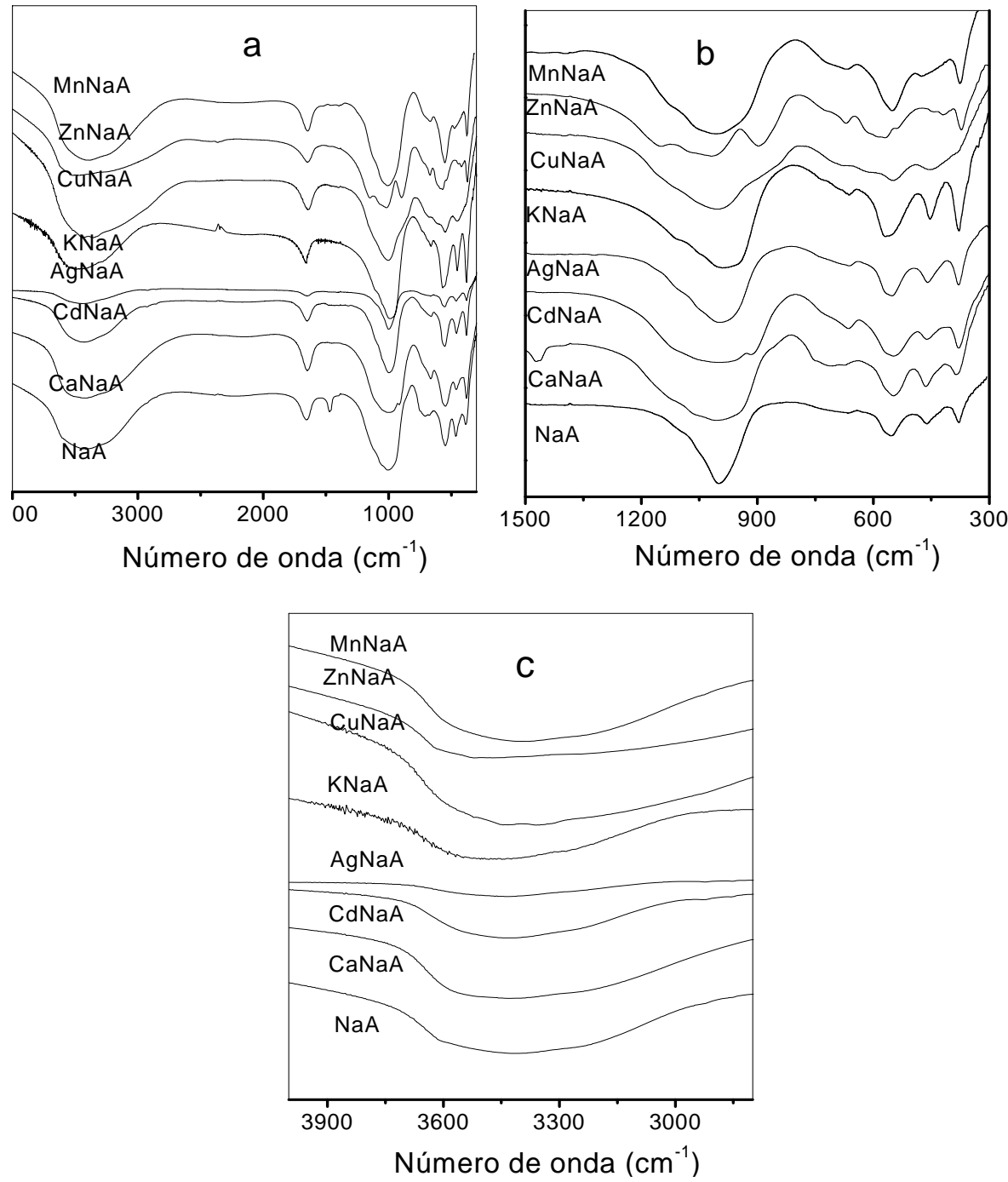

Figura 4: (a) Espectros IR de zeolitas LTA intercambiadas; (b) Vibraciones del enrejado de las zeolitas y (c) vibraciones Si-OH de las zeolitas LTA estudiadas.

\subsubsection{Adsorción de nitrógeno}

Isotermas de adsorción de $\mathrm{N}_{2}$ a $76 \mathrm{~K}$ en zeolita LTA intercambiada y seleccionada son mostradas en la Figura 5 (presión relativa $p / p^{0}$ vs. volumen adsorbido por gramo de zeolita). La figura 6 muestra las isotermas de adsorción en escala logarítmica de $p / p^{0}$ para estas zeolitas en el intervalo de $p / p^{0}=10^{-7}-10^{0}$. Los ciclos 
de histéresis mostrados por las isotermas de estas zeolitas son del tipo H3, característicos para la existencia de poros en forma de rendija, IUPAC, 1985 [1]. La Tabla 2 muestra valores de parámetros texturales obtenidos a partir del análisis de las isotermas de adsorción, ejemplo de estos son: la superficie específica ( $A s$ ) obtenida por a) el método $\mathrm{BET}, A s_{B}$, b) la ecuación Langmuir, $A s_{L}$ y c) área externa evaluada por el método $t$, $A e$. En la misma tabla están dados otros parámetros muy importantes como d) la constante del método BET, $C_{B}$ y e) el volumen total de poros, $V_{\Sigma}$.

Tabla 2: Propiedades texturales de Zeolitas LTA.

\begin{tabular}{|c|c|c|c|c|c|c|c|}
\hline ZEOLITA & $\begin{array}{c}\mathrm{A}_{\mathrm{SL}} \\
\mathrm{m}^{2} \mathrm{~g}^{-1}\end{array}$ & $\begin{array}{c}\mathrm{A}_{\mathrm{SB}} \\
\mathrm{m}^{2} \mathrm{~g}^{-1}\end{array}$ & $\begin{array}{c}\text { Ae } \\
\mathrm{m}^{2} \mathrm{~g}^{-1}\end{array}$ & $\mathrm{C}_{\mathrm{B}}$ & $\begin{array}{c}\mathrm{V}_{\Sigma} \\
\mathrm{cm}^{3} \mathrm{~g}^{-1}\end{array}$ & $\begin{array}{c}\mathrm{W}_{0} \\
\mathrm{~cm}^{3} \mathrm{~g}^{-1}\end{array}$ & $\begin{array}{c}\mathrm{V}_{\text {meso }} \\
\mathrm{cm}^{3} \mathrm{~g}^{-1}\end{array}$ \\
\hline $\mathrm{NaA}$ & 18.23 & 12.60 & 9.070 & -302 & 0.029 & 0.0037 & 0.0260 \\
\hline AgNaA & 15.90 & 11.020 & 5.890 & $-3 \times 10^{3}$ & 0.027 & 0.0036 & 0.0234 \\
\hline KNaA & 19.70 & 12.97 & 10.59 & 12.850 & 0.003 & 0.0028 & 0.0002 \\
\hline $\mathrm{CuNaA}$ & 411.10 & 264.50 & 64.170 & -44.150 & 0.195 & 0.121 & 0.074 \\
\hline $\mathrm{ZnNaA}$ & 473.53 & 423.560 & 30.046 & -57.00 & 0.350 & 0.158 & 0.192 \\
\hline $\mathrm{CdNaA}$ & 498.50 & 315.39 & 32.02 & -37.03 & 0.206 & 0.163 & 0.043 \\
\hline $\mathrm{MnNaA}$ & 512.20 & 359.10 & 24.70 & -63.78 & 0.232 & 0.173 & 0.059 \\
\hline $\mathrm{CaNaA}$ & 545.30 & 361.73 & 118.0 & -75.88 & 0.244 & 0.151 & 0.093 \\
\hline
\end{tabular}

Donde: $\mathrm{A}_{\mathrm{SL}}$ es la superficie específica estimada por el método Langmuir, $\mathrm{A}_{\mathrm{SB}}$ es la superficie específica estimada por el método BET, Ae es la superficie externa utilizando el método $t, \mathrm{C}_{\mathrm{B}}$ es la constante BET, $\mathrm{V}_{\Sigma}$ es el volumen total de poros, $\mathrm{W}_{0}$ es el volumen de microporos evaluado por el método $\alpha_{\mathrm{s}}$ de Sing y $\mathrm{V}_{\text {meso }}$ es calculado por la substracción de $\mathrm{V}_{\Sigma}-\mathrm{W}_{0}$

La alta capacidad de adsorción de la zeolita intercambiada con cationes bivalentes, figura 5a, con respecto a las isotermas de las zeolitas monovalentes, figura 5b, es notoria. Las isotermas de adsorción para las zeolitas LTA estudiadas presentan características típicas. Todas las isotermas de adsorción correspondientes a los cationes bivalentes corresponden al tipo I puro de acuerdo a la clasificación de la IUPAC, mientras que las isotermas correspondientes a los cationes monovalentes son del tipo II-IV, dentro de esta misma clasificación. Para la gran mayoría de las zeolitas analizadas, la constante $C_{B}$ de la ecuación BET es negativa indicando que el método BET es inoperante debido a que el fenómeno de formación de multicapas no es posible que se realice en poros de dimensiones moleculares. El plato o zona de saturación en estas zeolitas se forma en la zona de $p / p^{0}$ = 0.1- 0.8. Por otra parte, las isotermas correspondientes a las zeolitas CuNaA, MnNaA y CdNaA manifiestan a $p / p^{0}=0.4$ un rompimiento abrupto de la capa adsorbida atribuido al efecto tensil, Gregg y Sing, 1982 [1] ] El intervalo de $p / p^{0}$ usado para calcular los parámetros de la ecuación BET estuvo en el orden de $0.04-0.2$. De los valores de superficie específica evaluada por la ecuación Langmuir $\left(\mathrm{As}_{\mathrm{L}}\right)$ se establece la siguiente secuencia para las zeolitas LTA obtenidas: $\mathrm{CaNaA}>\mathrm{MnNaA}>\mathrm{CdNaA}>\mathrm{ZnNaA}>\mathrm{CuNaA}>\mathrm{NaA}>\mathrm{KNaA}$. Esta secuencia sigue un comportamiento similar cuando se comparan los parámetros característicos para sólidos microporosos (i.e. área externa, Ae y volumen de microporos, $\mathrm{W}_{0}$ ). Los resultados mostrados anteriormente indican que los procesos de intercambio iónico con sales bivalentes se ven favorecidos al lograrse un incremento en las propiedades texturales. 

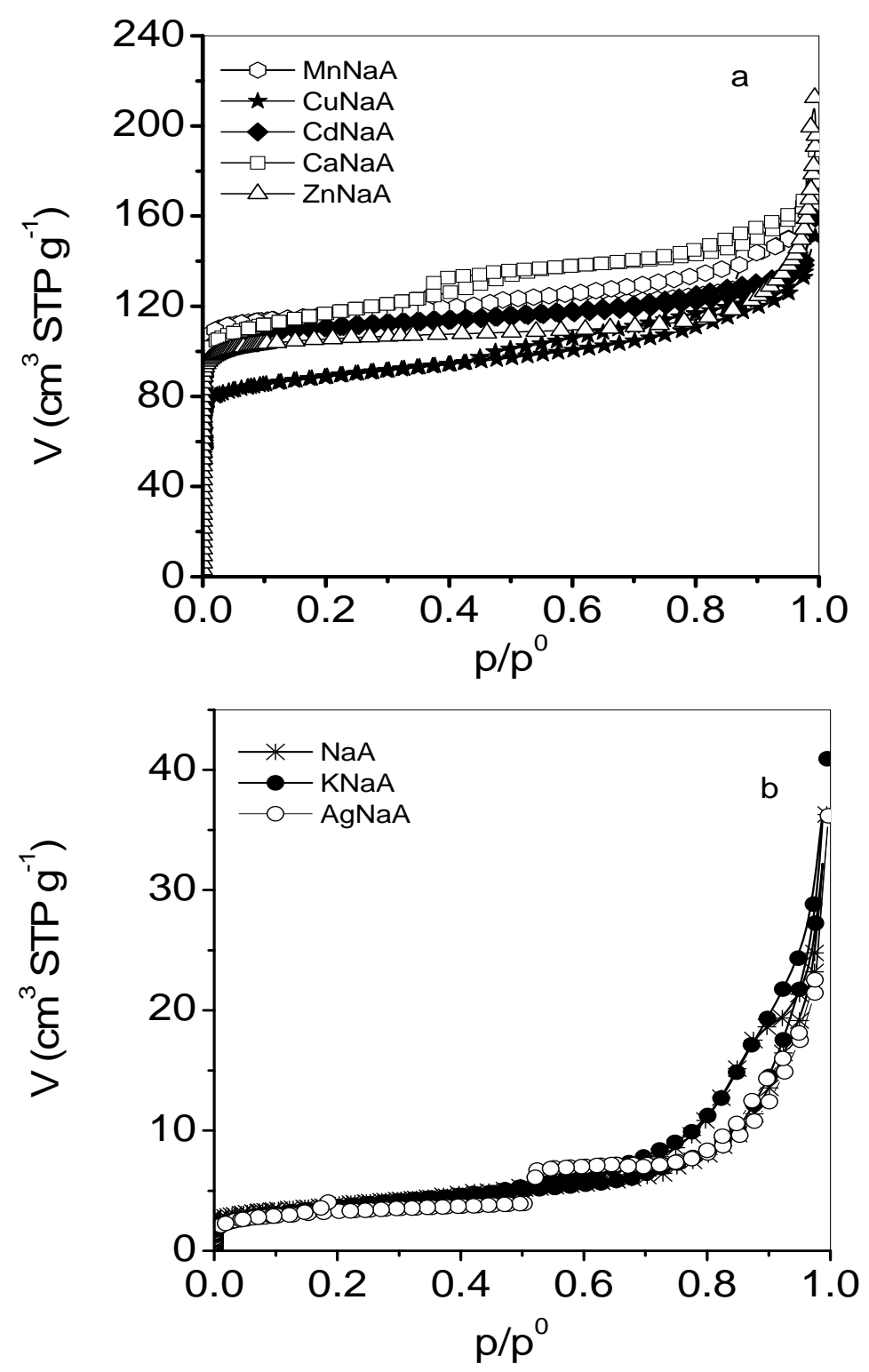

Figura 5: Isotermas de adsorción de $\mathrm{N}_{2}$ a $76 \mathrm{~K}$ en zeolitas LTA. a) cationes bivalentes y b)cationes monovalentes.

Los sitios SI son preferencialmente ocupados seguidos de los sitios SII, y los sitios SIII solamente son llenados después de que todos los sitios SI y SII han sido ocupados. En la forma sódica (NaA) existen doce cationes por caja. Estos cationes se pueden acomodar en los ocho sitios tipo SI y los tres sitios SII, con un catión en un sitio SIII. Todas las ventanas están obstruidas parcialmente por los $\mathrm{Na}^{+}$(radio atómico $=1.90 \AA$ ) y la apertura efectiva de esta ventana es reducida de 4.40 a $3.80 \AA$ Si los cationes $\mathrm{Na}^{+}$son intercambiados por cationes bivalentes $\left(\mathrm{M}^{2+}\right)$ el número de cationes por celda disminuye. Cuando se realiza al menos el $67 \%$ de intercambio, existen solamente ocho cationes por celda y todos estos pueden ser acomodados en los sitios SI. Así, en la forma $\mathrm{Ca}^{2+}$ (radio atómico $=1.97 \AA$ ) o $\mathrm{Mn}^{2+}$ (radio atómico = $1.26 \AA$ ) la apertura efectiva es incrementada y algunas moléculas relativamente grandes pueden penetrar. Por el contrario, considerando que el diámetro de los $\mathrm{K}^{+}$(radio atómico $=2.35 \AA$ ) es mayor que el diámetro de los $\mathrm{Na}^{+}$, un tamiz con una apertura efectiva (tamiz 3A) es obtenido por el intercambio con $\mathrm{K}^{+}$, Chintansinh, 2004 [5]]. 
La irregularidad en algunos de los valores obtenidos puede obedecer a que los cationes que se encuentran en la microporosidad o en las posiciones I, II o III no fueron intercambiados en su totalidad, o si lo fueron, éstos quedaron excluidos a causa de que las ventanas de entrada a los microporos se obstruyeron por las "sales" que se formaron durante el proceso de intercambio iónico.

El proceso de llenado de ultramicroporos en la zeolita $\mathrm{ZnNaA}$ es evidente cuando se observa un incremento en la cantidad de sustancia adsorbida que ocurre a muy bajas presiones relativas en la isoterma de adsorción de $\mathrm{N}_{2}$ de alta resolución, Cejka et al., 2004 [] ] mostrada en la Figura 6. En esta misma figura se pone de manifiesto la existencia de un proceso de llenado de microporos secundario, observado por la aparición de una curvatura en la zona de $p / p^{0}=10^{-5}$. Esta curvatura también puede ser relacionada al fenómeno de multicapas de adsorción que se esta realizando en los mesoporos y área externa de la zeolita. En esta isoterma de alta resolución se observan los distintos mecanismos de adsorción que ocurren en la adsorción de $\mathrm{N}_{2}$ a $76 \mathrm{~K}$.

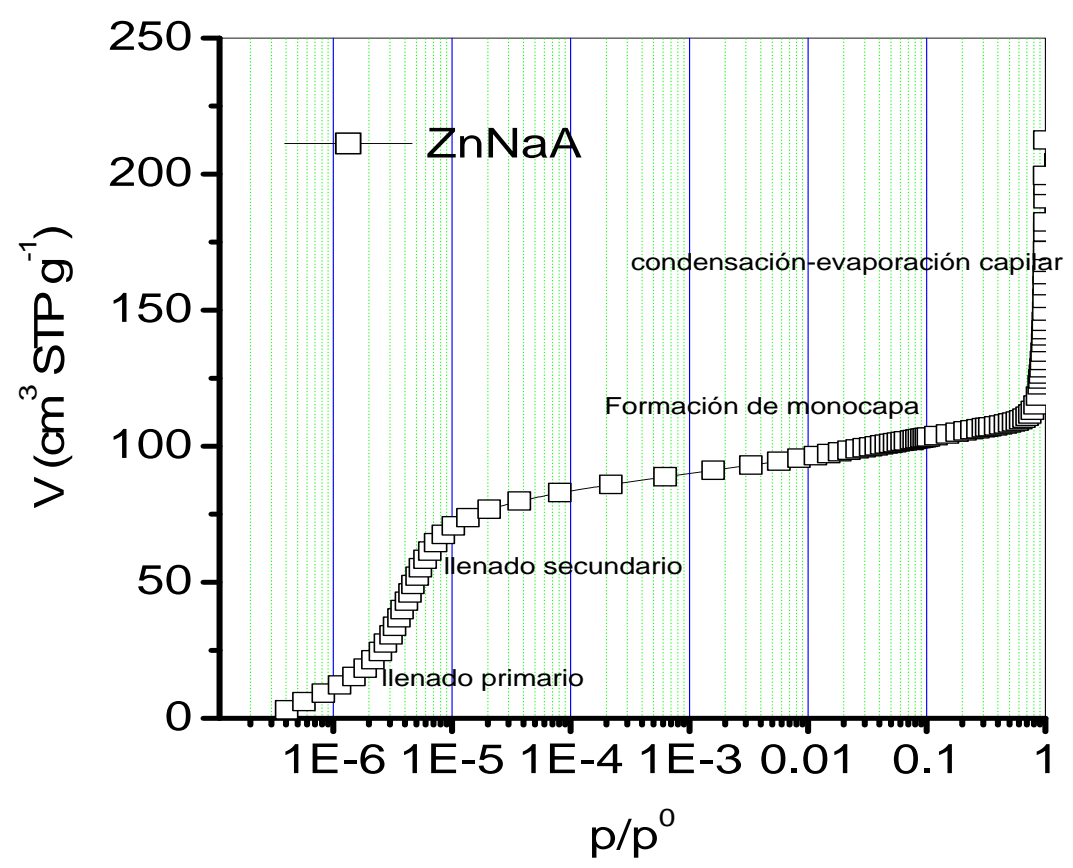

Figura 6: Isotermas de adsorción de $\mathrm{N}_{2}$ a $76 \mathrm{~K}$ de alta resolución en zeolitas $\mathrm{ZnNaA}$.

\subsubsection{Microporosidad}

El volumen total de microporos $\left(W_{0}\right.$, en $\left.\mathrm{cm}^{3} \mathrm{~g}^{-1}\right)$ de la zeolita LTA intercambiada están listados en la Tabla 1. Estos valores han sido calculados a partir de los gráficos $\alpha_{\mathrm{s}}$. Los valores del volumen de microporos reportados en este trabajo son similares a los valores reportados obtenidos por el método $\alpha_{s}$ con zeolitas mordenitas, Hernández et al., 2005 [17] y clinoptilolitas, Hernández et al., 2005 [18].

En los gráficos $\alpha_{\mathrm{s}}$ se consideró el intervalo comprendido entre 0.05 a 2.4, figura 7a y de 0.05-0.4 para los gráficos de alta resolución, figura 7b. Para la construcción de los gráficos $\alpha_{\mathrm{s}}$ fueron usados los valores experimentales de un sólido de referencia llamado $\alpha$-cuarzo. La selección del $\alpha$-cuarzo como material de referencia se baso en el hecho de que el proceso de adsorción en este sólido ocurre de manera similar a la de una superficie abierta y no hay acceso a microporos.

El proceso de llenado de microporos (ultramicroporos y supermicroporos) puede ser evidenciado a través del análisis de los gráficos $\alpha_{\mathrm{s}}$ de alta resolución, iniciando a muy bajas $p / p^{0}\left(10^{-5}\right)$, Figura 6 . De esta figura se observa que existen diferencias muy marcadas en la forma de estos gráficos, principalmente en las zeolitas intercambiadas con los cationes bivalentes con respecto a las zeolitas NaA y KNaA muy probablemente debido a que los tamaños de estos cationes son mayores que los demás como se muestra en la tabla 1 . Se puede observar una distorsión muy pronunciada a valores muy bajos de $p / p^{0}$, lo cual puede ser atribuido a una 
interacción adsorbato-adsorbente en poros de dimensiones moleculares, es decir, el proceso de llenado de microporos que esta sucediendo en los ultramicroporos (poros con amplitud inferior a 3.5 Å), Kaneko, 1997 [19]. Este tipo de gráficos $\alpha_{\mathrm{s}}$ son típicos para adsorbentes con microporos que presentan un estrecho intervalo de tamaño de poros y proporcionan dos o más etapas del llenado de microporos. De la figura $7 \mathrm{~b}$ se observa el establecimiento de tres zonas: la zona III, $\alpha_{s}>0.4$, corresponde a la adsorción en los mesoporos y área externa de la zeolita en estudio. La extrapolación a la ordenada en $p / p^{0}=0$ permite el cálculo del volumen de microporo total $W_{0}$. La zona II, $\alpha_{\mathrm{s}}=0.4-0.05$, ha sido creada por la remoción parcial de la zeolita matriz (NaA) de los constituyentes originales de la síntesis o se hacen accesibles estas entidades debido al tratamiento de intercambio iónico. La zona I, $\alpha_{\mathrm{s}}<0.05$, relaciona la etapa inicial del llenado inicial de microporos más angostos (los más energéticos) o las constricciones. La diferencia entre las figuras 6 y 7 es que en esta última se observa con toda claridad el proceso del llenado de los ultramicroporos, supermicroporos y mesoporos existentes en la estructura de la zeolita ZnNaA, mostrando a muy bajas presiones relativas la interacciòn electròstatica entre los cationes de nitrògeno y zinc. Al principio de la isoterma se nota una fuerte interacción que tiene lugar en los microporos de menor tamaño debido a que en esa zona los campos electrostáticos son mayores.
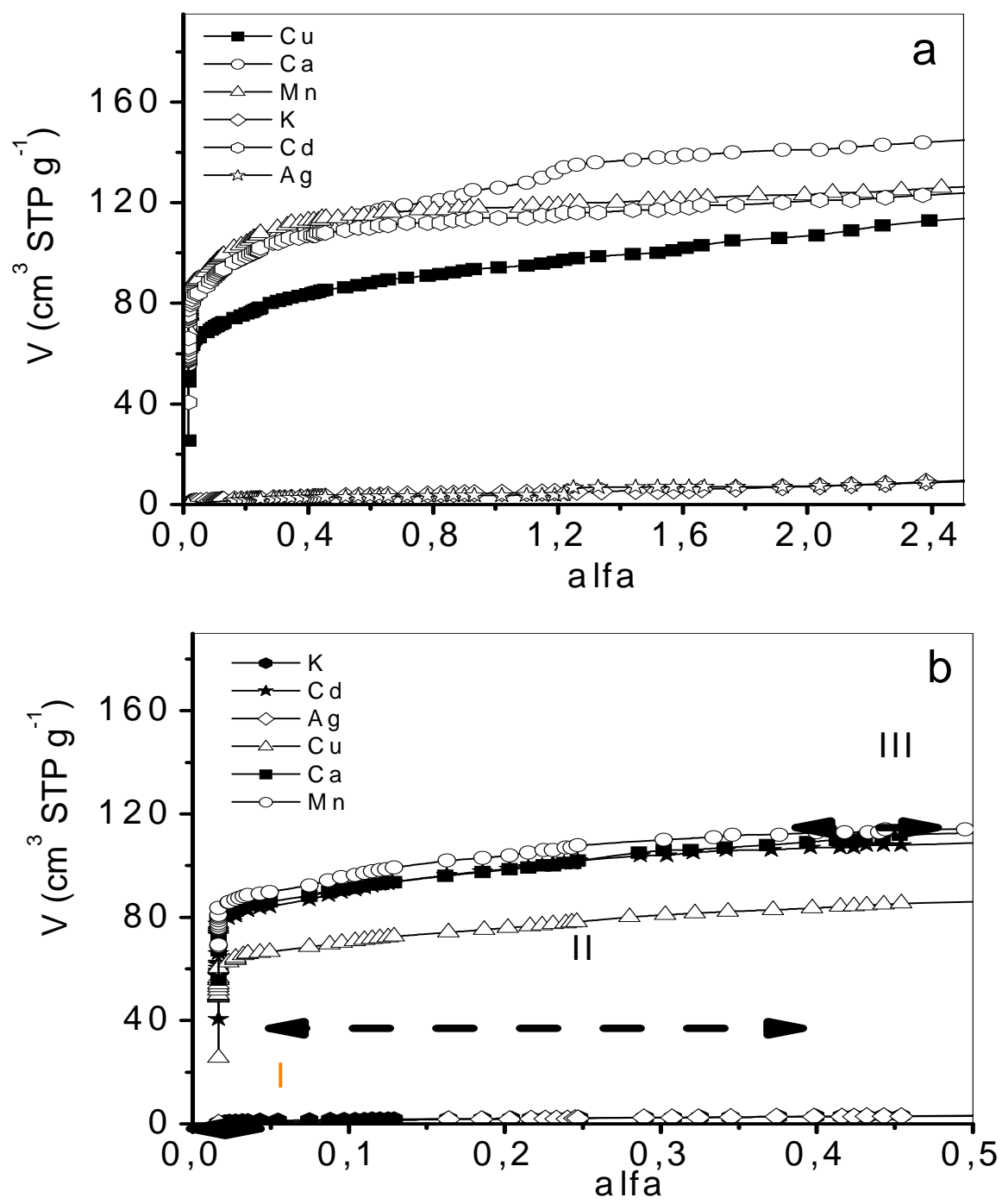

Figura 7: Gráficos $\alpha_{s}$ en zeolitas LTA. a) Normal, b) Alta resolución 


\section{CONCLUSIONES}

La síntesis de zeolitas LTA y sus intercambios con distintas sales fueron estudiadas. Distintos parámetros sobre sus propiedades de textura han sido contemplados. Los patrones de difracción de Rayos X de las zeolitas LTA obtenidas presentan patrones característicos para este tipo de zeolitas. Los estudios por microscopía electrónica de barrido muestran un gran grupo de cristales pequeños, su existencia proporciona la ventaja de tener una gran área externa y pueden reducir la resistencia a la transferencia a la masa. Los estudios IR de las zeolitas LTA muestran que las señales prominentes de este tipo de materiales exhiben las vibraciones de tensión de los puentes de $\mathrm{H}$ entre el agua adsorbida físicamente que se encuentra en las cavidades y los oxígenos superficiales así como las vibraciones de tensión y deformación de los H-O-H correspondientes al agua molecular adsorbida. Las zeolitas LTA obtenidas describen porosidad alta la cual es influida por los tratamientos de intercambio iónico, sobre todo, cuando se realiza con cationes bivalentes. La presencia de este tipo de cationes en los sitios SI aumenta su capacidad de adsorción haciendo accesible el volumen de microporos y sus áreas externas son considerables. Las contribuciones de los ultramicroporos y supermicroporos al volumen de microporo total en las zeolitas LTA puede ser evaluado a través de los gráficos $\alpha_{\mathrm{s}}$ de alta resolución. Las isotermas de adsorción de $\mathrm{N}_{2}$ a $76 \mathrm{~K}$ describen isotermas tipo I indicando la participación tanto de microporos (especialmente supermicroporos) y mesoporos.

\section{AGRADECIMIENTOS}

Se agradece el apoyo financiero al CONACyT a través de los proyectos 47631-F y 76739.

\section{BIBLIOGRAFÍA}

[1] SING, K.S.W., EVERETT, D.H., HAUL, R.A.W., MOSCOU, L., PIERROTTI, R., ROUQUEROL, J., SIEMIENIESWKA, T., "Presentación de datos de fisisorción en sistemas gas/sólido", Pure Applied Chemical, v. 85, n. 57, pp. 603-619, January 1985.

[2] CURTIS, R.A., DEEM, M.W., “A Statistical mechanics study of ring size, ring shape, and the relation to pores found in zeolites”, Journal Physic Chemistry. B, v. 107, n. 33, pp. 8612-8620, July 2003.

[3] CHANG, H.L., SHIH, W.H., "Synthesis of zeolites A and X from fly ashes and their ion-exchange behavior with cobalt ions”, Industrial Engineering Chemistry Research, v. 39, n. 11, pp. 4185-4191, August 2000 .

[4] HERNÁNDEZ, M. A., Determinación Experimental de la Textura en Sólidos Microporosos de Naturaleza Zeolítica, Thesis Ph. D., Universidad Autónoma Metropolitana, México, 2000.

[5] CHINTANSINH, D.CH., JINCE, S., RAKSH V.J., "Pore-size engineering of zeolite A for the size/shape selective molecular separation”, Industrial Engineering Chemistry Research, v. 44, n. 6, pp. 1780-1786, December 2005.

[6] CEJKA, J., ZILKOVA, N., RATHOUSKY, J., ZUKAL, A., JAGIELLO, J., "High-resolution adsorption of nitrogen on mesoporous alumina” Langmuir, v. 20, n.1, pp. 7532-7539, June 2004.

[7] RIBEIRO CARROTT, M.M.L., RUSSO, P.A., CARVAlHAL, C., CARROTT, P.J.M., MARQUES, P., LOPES, J.M., GENER, I., GUISNET, M., RIBEIRO FR., “Adsorption of n-pentane and iso-octane for evaluation of the porosity of dealuminated BEA zeolites”, Microporous and Mesoporous Materials, v. 81, n. 1-3, pp. 259-267, June 2005.

[8] SING, K.S.W., "Characterization of porous materials: past, present and future”, Coloids and Surfaces APhysicochemical and Engineering Aspects, v. 241, n. 1-3, pp. 3-7, July 2004.

[9] JARONIEC, M., KRUK, M.,OLIVIER, J.P. "Standard nitrogen adsorption data for characterization of nanoporous silicas”, Langmuir, v. 15, n. 16, pp. 5410-5413, April 1999. 
[10] ROBERTS, R.A., SING, K.S. W., TRIPATHI, V., “Adsorption of nitrogen and neopentane vapor by microporous carbons”, Langmuir, v. 3, n. 3, pp. 331-335, May-June 1987.

[11] REMY, M.J., PONCELET, P.A., "New approach to the determination of the external surface and micropore volume of zeolites from the nitrogen adsorption isotherm at 77 K”, Journal Physic Chemistry, v. 99, n. 2, pp. 773-779, January 1995.

[12] ARMATAS, G.S., PETRAKIS, D.E., POMONIS, P.J., “ A method of distinction between micrporosity and mesoporosity using BET-scatchard plots”, Microporous and Mesoporous Materials, v. 83, n. 1-3, pp. 251-161, September 2005.

[13] RUTHVEN, D.M., KAUL, B.K., “Adsorption of aromatics hydrocarbons in NaX zeolite. 1. equilibrium”, Industrial Engineering Chemistry Research, v. 32, n. 9, pp. 2047-2052, September 1993.

[14] HUAI, Y.Z., PEGIE C., ETIENNE, F.V., BAO, L.S., XUEPING, G., "Quantitative information on pore size distribution from the tangents of comparison plots”, Langmuir, v. 20, n. 23, pp. 10115-10122, August 2004.

[15] HUDEC, P., SMIESKOVA, P., ZIDEK, Z., SCHNEIDER, P., SOLCOVA, O., "Determination of microporous structure of zeolites by t-plot method". State of the Art. In: Impact of Zeolites and Other Porous Materials on the New Technologies at the Beginning of the new millennium, pts A and B,1 ed. (pp. 1587- 1594), Amsterdam, Elsevier, 2002.

[16] GREG, S.J., SING, K.S.W., Adsorption surface area and porosity, 2 ed. London, Academic Press, 1982.

[17] HERNÁNDEZ, M.A., PETRANOVSKII, V., AVALOS, M., PORTILlO, R., ROJAS, F., LARA, V.H., "Influence of the $\mathrm{Si} / \mathrm{Al}$ framework ratio on the microporosity of dealuminated mordenite as determined from $\mathrm{N}_{2}$ adsorption”, Separation Science and Technology, v. 41, n. 9, pp. 1907-1925, July 1906.

[18] HERNANDEZ, M.A., CORONA, L., GONZALEZ, A.I., ROJAS, F., SILVA, F., “A quantitative study of the adsorption of aromatic hydrocarbons (Benzene, Toluene, and p-Xylene) on dealuminated clinoptilolites”, Industrial Engineering Chemistry Research, v. 44, n. 9, pp. 2908-2916, March 2005.

[19] KANEKO, K., TAO, Y.S., KANOH, H., "Developments and structures of mesopores in alkaline-treated ZSM-5 zeolites”, Adsorption Journal of the International Adsorption Society, v. 12, n. 5-6, pp. 309-316, September 2006. 